\title{
Attenuation compensation for least-squares reverse time migration using the viscoacoustic-wave equation
}

\author{
Gaurav Dutta ${ }^{1}$ and Gerard T. Schuster ${ }^{1}$
}

\begin{abstract}
Strong subsurface attenuation leads to distortion of amplitudes and phases of seismic waves propagating inside the earth. Conventional acoustic reverse time migration (RTM) and least-squares reverse time migration (LSRTM) do not account for this distortion, which can lead to defocusing of migration images in highly attenuative geologic environments. To correct for this distortion, we used a linearized inversion method, denoted as $Q_{p}$-LSRTM. During the leastsquares iterations, we used a linearized viscoacoustic modeling operator for forward modeling. The adjoint equations were derived using the adjoint-state method for back propagating the residual wavefields. The merit of this approach compared with conventional RTM and LSRTM was that $Q_{p}$-LSRTM compensated for the amplitude loss due to attenuation and could produce images with better balanced amplitudes and more resolution below highly attenuative layers. Numerical tests on synthetic and field data illustrated the advantages of $Q_{p}$-LSRTM over RTM and LSRTM when the recorded data had strong attenuation effects. Similar to standard LSRTM, the sensitivity tests for background velocity and $Q_{p}$ errors revealed that the liability of this method is the requirement for smooth and accurate migration velocity and attenuation models.
\end{abstract}

\section{INTRODUCTION}

Fluids trapped in overburden structures cause strong attenuation of P-waves that can decrease the resolution of migration images. This can be attributed to the fact that the real earth is anelastic and therefore distorts the amplitudes and phases of the propagating seismic waves (Aki and Richards, 1980). If the subsurface attenuation is very strong, ignoring it during migration can lead to blurring of migration amplitudes below these layers.

Attenuation of P-waves can be quantified by a quality factor $Q_{p}$ which accounts for the phase shift as a function of the frequency content of the propagating waves and the distance traveled. Lower values of $Q_{p}$ imply more energy loss of the wave per cycle or high attenuation. For example, the values of $Q_{p}$ for unconsolidated gassandstones and shales are typically very low $\left(Q_{p} \approx 15-30\right)$, which necessitates the need to account for $Q_{p}$ during imaging. Another example is the presence of gas pockets in the North Sea sediments that distort the migrated amplitudes of the underlying reflectors.

The earliest efforts at compensating for attenuation loss in seismic data were performed in the data domain using an inverse $Q_{p}$-filtering method (Bickel and Natarajan, 1985; Hargreaves and Calvert, 1991). These data domain methods partially correct for the attenuation loss because attenuation loss occurs during wave propagation, and therefore, $Q_{p}$ compensation is required during migration.

For prestack depth migration, Xin et al. (2008) and Xie et al. (2009) perform attenuation compensation using ray-tracing methods. Dai and West (1994), Yu et al. (2002), Wang (2008), and Valenciano et al. (2011) use one-way wave-equation migration methods in the frequency domain for attenuation compensation. The more accurate two-way wave-equation migration or reverse time migration (RTM) (Baysal et al., 1983; McMechan, 1983; Whitmore, 1983) does not take into account attenuation because the lossless acoustic-wave equation is used for wavefield extrapolation. Similarly, for least-squares reverse time migration (LSRTM) (Plessix and Mulder, 2004; Dai and Schuster, 2010; Dai et al., 2011; Wong et al., 2011), if the gradient computation at each iteration is done by RTM of the data residuals, then the reflector image below the attenuation zone will be degraded in quality.

In the context of RTM, Zhang et al. (2010) use the dispersion relation for a viscoacoustic medium (Kjartansson, 1979) and derive a pseudodifferential equation with separate controls over phase and amplitude to model and migrate viscoacoustic waves. They require

\footnotetext{
Manuscript received by the Editor 10 November 2013; revised manuscript received 20 May 2014; published online 1 October 2014.

${ }^{1}$ King Abdullah University of Science and Technology, Department of Earth Science and Engineering, Thuwal, Saudi Arabia. E-mail: gaurav.dutta@kaust.edu .sa; gerard.schuster@kaust.edu.sa.

(C) 2014 Society of Exploration Geophysicists. All rights reserved.
} 
a regularization process to stabilize the back-propagating wavefield because the high-frequency amplitudes increase with time and lead to numerical instability. Suh et al. (2012) extend the work of Zhang et al. (2010) for a VTI medium in which they ignore the phase effects due to attenuation and only compensate for the amplitude loss. Their approach suffers from the same instability problem during back propagating the receiver wavefield as in Zhang et al. (2010). They apply a high-cut filter to the receiver wavefield to stabilize the amplification of high-frequency components. Bai et al. (2013) derive a new viscoacoustic wave equation without any memory variable. They account for attenuation by incorporating a pseudodifferential operator in the time and space domains. Similar to Zhang et al. (2010) and Suh et al. (2012), they apply a high-cut filter to stabilize the wavefield propagation. Fletcher et al. (2012) propose a stable approach in which they apply separate phase and amplitude filters to the source and receiver wavefields to compensate for amplitude and phase effects. These filters are applied prior to imaging and are estimated based upon running acoustic and viscoacoustic propagators twice to estimate attenuated amplitudes along wavepaths.

In this paper, we use the standard time-domain viscoacoustic wave equation for wavefield extrapolation and use the linearized least-squares inversion method to compensate for the attenuation loss. For least-squares migration, a linearized viscoacoustic waveequation modeling operator based on the perturbation of the bulk modulus is derived, and then the appropriate adjoint equations and imaging condition are used for the least-squares iterations. The advantage of this approach over other existing methods is that we do not need to modify and regularize the adjoint wave equations during the receiver-side wavefield extrapolation to compensate for the attenuation loss, and hence, it is always stable. Accounting for $Q_{p}$ in the modeling and adjoint operations coupled with the least-squares iterations compensates for the amplitude loss due to attenuation. Numerical tests on synthetic and field data show that the amplitudes below high-attenuative layers are better balanced in the inverted images from $Q_{p}$-LSRTM compared with standard RTM and LSRTM, and the reflectors are focused at the right locations. The disadvantage is that this method is expensive (computational cost per iteration is more than six times that of standard RTM), and the computational cost becomes proportional to the number of least-squares iterations. However, similar to standard LSRTM, the cost of $Q_{p}$-LSRTM can be significantly reduced by using a multisource-encoded strategy (Dai and Schuster, 2009; Tang, 2009; Dai et al., 2010; Huang and Schuster, 2012). Another liability of this method is that besides the input requirement of a smooth and accurate migration velocity model, a good estimate of the $Q_{p}$ model is also needed.

This article is organized into five sections. After the introduction, the second section describes the theory of standard LSRTM, and then the time-domain implementation of $Q_{p}$-LSRTM is presented in the third section. Numerical results on synthetic and field data are presented in the fourth section. Discussions based on the numerical simulations are in the fifth section and the conclusions are in the last section.

\section{THEORY OF ACOUSTIC LEAST-SQUARES REVERSE TIME MIGRATION}

Conventional least-squares migration seeks to reconstruct the earth's reflectivity image from the recorded waveform data under the Born approximation (Lailly, 1984; Tarantola, 1984; Nemeth et al., 1999; Duquet et al., 2000; Plessix and Mulder, 2004; Wong et al., 2011; Dai et al., 2012). In this section, we will briefly review the theory of acoustic LSRTM in the time-domain.

For a given background velocity model $v_{0}(\mathbf{x})$, the pressure wavefield $p_{0}(\mathbf{x}, t)$ satisfies the acoustic-wave equation with constant density:

$$
\frac{1}{v_{0}(\mathbf{x})^{2}} \frac{\partial^{2} p_{0}(\mathbf{x}, t)}{\partial t^{2}}-\nabla^{2} p_{0}(\mathbf{x}, t)=f\left(\mathbf{x}_{s}, t\right)
$$

where $f\left(\mathbf{x}_{s}, t\right)$ represents a band-limited point source function at $\mathbf{x}=\mathbf{x}_{s}$.

For LSRTM, we seek to find the perturbation in the wavefield $\delta p(\mathbf{x}, t)$ related to the perturbation in the velocity $\delta v(\mathbf{x})$. Under the Born approximation, the perturbed wavefield can be calculated as (Stolt and Benson, 1986; Plessix and Mulder, 2004; Dai et al., 2012)

$$
\frac{1}{v_{0}(\mathbf{x})^{2}} \frac{\partial^{2} \delta p(\mathbf{x}, t)}{\partial t^{2}}-\nabla^{2} \delta p(\mathbf{x}, t)=\frac{m(\mathbf{x})}{v_{0}(\mathbf{x})^{2}} \frac{\partial^{2} p_{0}(\mathbf{x}, t)}{\partial t^{2}}
$$

where the reflectivity image $m(\mathbf{x})$ is defined as $m(\mathbf{x})=\frac{2 \delta v(\mathbf{x})}{v_{0}(\mathbf{x})}$. To find the perturbed wavefield numerically, two finite-difference simulations are required, one for the background wavefield $p_{0}(\mathbf{x}, t)$ given by equation 1 and the other for the perturbed wavefield $\delta p(\mathbf{x}, t)$ given by equation 2 . In the context of least-squares migration, the solution to equation 2 can be represented as the matrixvector operation $\mathbf{d}=\mathbf{L m}$, where $\mathbf{d}$ is the data, $\mathbf{L}$ is a linear modeling operator, and $\mathbf{m}$ represents the migration image which in this case is related to the perturbation in velocity.

The receiver-side wavefield $r(\mathbf{x}, t)$ can be computed by reverse time propagation proposed by McMechan (1983) and Whitmore (1983) and is equivalent to the adjoint state solution defined by Lailly (1984) and Plessix (2006). The back-projected field $r(\mathbf{x}, t)$ is the solution to

$$
\frac{1}{v_{0}(\mathbf{x})^{2}} \frac{\partial^{2} r(\mathbf{x}, t)}{\partial t^{2}}-\nabla^{2} r(\mathbf{x}, t)=d\left(\mathbf{x}_{g}, t ; \mathbf{x}_{s}\right),
$$

where $d\left(\mathbf{x}_{g}, t ; \mathbf{x}_{s}\right)$ represents the recorded receiver data at a geophone location, $\mathbf{x}=\mathbf{x}_{g}$ for a source at $\mathbf{x}=\mathbf{x}_{s}$. The migration image $m(\mathbf{x})$ can be computed by taking a zero-lag crosscorrelation of the source and receiver wavefields as

$$
m(\mathbf{x})=\sum_{t} \frac{1}{v_{0}(\mathbf{x})^{2}} \frac{\partial^{2} p_{0}(\mathbf{x}, t)}{\partial t^{2}} r(\mathbf{x}, t)
$$

In matrix-vector notation, equation 4 is equivalent to the operation $\mathbf{m}=\mathbf{L}^{T} \mathbf{d}$. The reflectivity distribution $m(\mathbf{x})$ can be iteratively estimated using any gradient-based method such as the steepest descent method (Nemeth et al., 1999):

$$
\begin{aligned}
& \mathbf{g}^{(i)}=\mathbf{L}^{T}\left[\mathbf{L} \mathbf{m}^{(i)}-\mathbf{d}^{\mathrm{obs}}\right] \\
& \alpha=\frac{\left(\mathbf{g}^{(i)}\right)^{T} \mathbf{g}^{(i)}}{\left(\mathbf{L} \mathbf{g}^{(i)}\right)^{T}\left(\mathbf{L} \mathbf{g}^{(i)}\right)} \\
& \mathbf{m}^{(i+1)}=\mathbf{m}^{i}+\alpha \mathbf{g}^{(i)}
\end{aligned}
$$


where $i$ represents the iteration index for the least-squares inversion and $\mathbf{g}^{(i)}$ and $\alpha$ represent the gradient and the step-length, respectively. The gradient at each iteration is computed by RTM of the data residuals, $\mathbf{L m}^{(i)}-\mathbf{d}^{\text {obs }}$. In LSRTM, the operators, $\mathbf{L}$ and $\mathbf{L}^{T}$ depend on the background velocity model $v_{0}(\mathbf{x})$, which remains fixed at every iteration.

The theory of time-domain $Q_{p}$-LSRTM in now formulated in the next section.

\section{LEAST-SQUARES REVERSE TIME MIGRATION USING THE VISCOACOUSTIC-WAVE EQUATION}

For a viscoacoustic medium, the stress-strain relation is given by (Christensen, 1982; Carcione et al., 1988)

$$
\sum_{k=0}^{m} c_{k} \frac{\partial^{k} P}{\partial t^{k}}=\sum_{k=0}^{m} d_{k} \frac{\partial^{k} e}{\partial t^{k}},
$$

where $P$ denotes the pressure field, $e$ denotes the trace of the strain tensor matrix, $\partial^{k} / \partial t^{k}$ represents the $k$ th order time derivative, and $c_{k}$ and $d_{k}$ are coefficients related to the material properties of the medium. The pressure field can be expressed explicitly from equation 6 using the Laplace transform method as (Carcione et al., 1988)

$$
\begin{aligned}
P(t)= & -M_{R} \int_{-\infty}^{t} \dot{e}(\tau)[1 \\
& \left.-\sum_{l=1}^{L}\left(1-\frac{\tau_{\epsilon l}}{\tau_{\sigma l}}\right) \exp \left(-\frac{t-\tau}{\tau_{\sigma l}}\right)\right] \mathrm{d} \tau,
\end{aligned}
$$

where $\tau_{\sigma l}$ and $\tau_{\epsilon l}$ denote material relaxation times for the $l$ th mechanism, $L$ is the number of relaxation mechanisms for a standard linear solid model, and $M_{R}$ is the relaxed modulus of the medium. The equation of motion in an anelastic medium can be written as

$$
-\frac{1}{\rho} \nabla P=\frac{\partial \mathbf{v}}{\partial t}
$$

where $\rho$ represents the density $P$ represents the pressure wavefield, and $\mathbf{v}$ represents the particle velocity vector. Equations 7 and 8 together describe the deformation in a viscoacoustic medium.

Equation 7 is expensive to solve by numerical modeling because of the associated convolution operation. Robertsson et al. (1994) simplify the convolution term by introducing a memory variable term $r_{p}$. Blanch et al. (1995) later show that only one relaxation mechanism $(L=1)$ is sufficient for practical purposes. Thus, for practical numerical modeling applications, the equations of motion for a 2D viscoacoustic medium can be written as (Christensen, 1982; Carcione et al., 1988; Blanch and Symes, 1995)

$$
\begin{aligned}
& \frac{\partial P}{\partial t}+K \frac{\tau_{\epsilon}}{\tau_{\sigma}}(\nabla \cdot \mathbf{v})+r_{p}=f\left(\mathbf{x}_{s}, t\right), \\
& \frac{\partial \mathbf{v}}{\partial t}+\frac{1}{\rho} \nabla P=0 \\
& \frac{\partial r_{p}}{\partial t}+\frac{1}{\tau_{\sigma}}\left(r_{p}+K\left(\frac{\tau_{\epsilon}}{\tau_{\sigma}}-1\right)(\nabla \cdot \mathbf{v})\right)=0 .
\end{aligned}
$$

Here, $\mathbf{v}=\left\{v_{x}, v_{y}, v_{z}\right\}$ represents the particle velocity vector, $K$ represents the bulk modulus of the medium, and $f\left(\mathbf{x}_{s}, t\right)$ represents a band-limited point source function at $\mathbf{x}=\mathbf{x}_{s}$. The stress and strain relaxation parameters, $\tau_{\sigma}$ and $\tau_{\epsilon}$, are related to the quality factor $Q_{p}$ and the reference angular frequency $\omega$ (usually chosen to be the central frequency of the source wavelet) as (Robertsson et al., 1994)

$$
\tau_{\sigma}=\frac{\sqrt{1+\frac{1}{Q_{p}^{2}}}-\frac{1}{Q_{p}}}{\omega}, \quad \tau_{\epsilon}=\frac{1}{\omega^{2} \tau_{\sigma}} .
$$

Figure 1a and 1c shows the effect of attenuation on amplitude and phase of a propagating seismic wave in a homogeneous medium with a background velocity of $3000 \mathrm{~m} / \mathrm{s}$ and for different values of $Q_{p}$. A Ricker wavelet with $20-\mathrm{Hz}$ peak frequency is used as the source wavelet. The source is excited at the center of the model, and the snapshots are taken at different intervals of time. Figure $2 \mathrm{a}$ and $2 b$ compares the depth slices at different instances of time. It is evident from these figures that, as the wave starts propagating, the amplitudes in the three cases are very similar. However, as the propagation time increases and the wave travels a greater distance, the wave amplitude in case of $Q_{p}=20$ is attenuated the strongest. The high-frequency components are distorted more compared with the low frequencies. For moderate attenuation values $\left(Q_{p} \approx 40\right)$, as shown by the red curves in Figure $2 \mathrm{a}$ and $2 \mathrm{c}$, the attenuation effect at different times is still significant compared to the blue curves that represent the case in which there is no attenuation.

Similar to standard acoustic LSRTM, we seek to find the perturbed wavefield $\delta p(\mathbf{x}, t)$ related to the perturbation in the medium parameters. For algebraic simplicity, we make the following substitutions in equation 9:

$$
\tau=\frac{\tau_{\epsilon}}{\tau_{\sigma}}-1,
$$

so that equation 9 becomes
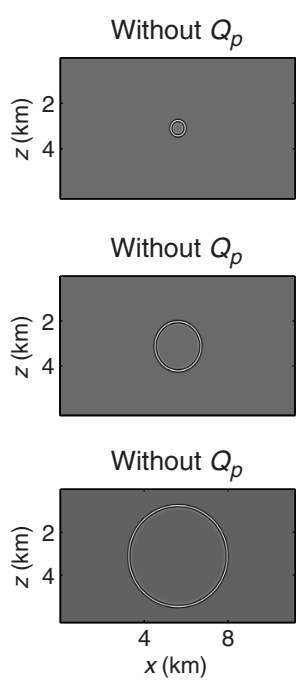
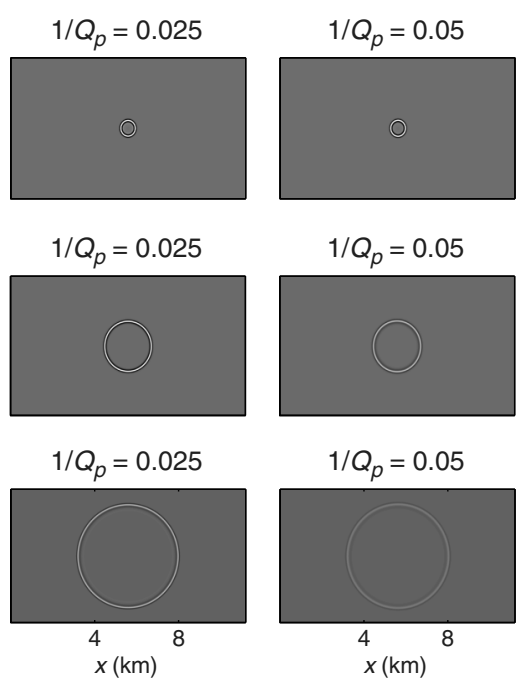

Figure 1. Snapshots showing an expanding wavefront at different instances of time for a medium with no attenuation (left panels), moderate attenuation (middle panels), and high attenuation (right panels). 


$$
\begin{aligned}
& \frac{\partial P}{\partial t}+K(\tau+1)(\nabla \cdot \mathbf{v})+r_{p}=f\left(\mathbf{x}_{s}, t\right), \\
& \frac{\partial \mathbf{v}}{\partial t}+\frac{1}{\rho} \nabla P=0, \\
& \frac{\partial r_{p}}{\partial t}+\frac{1}{\tau_{\sigma}}\left(r_{p}+\tau K(\nabla \cdot \mathbf{v})\right)=0 .
\end{aligned}
$$

Let $\rho_{0}, K_{0}, \tau_{\sigma_{0}}$, and $\tau_{0}$ be the background medium parameters. Perturbing them by an amount $\delta \rho, \delta K, \delta \tau_{\sigma}$, and $\delta \tau$, respectively, gives the new medium parameters as

a)

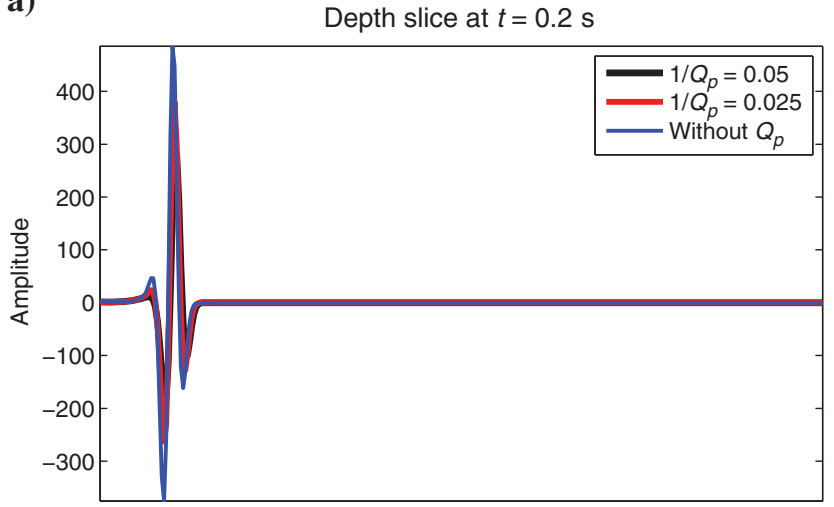

b)

Depth slice at $t=0.5 \mathrm{~s}$

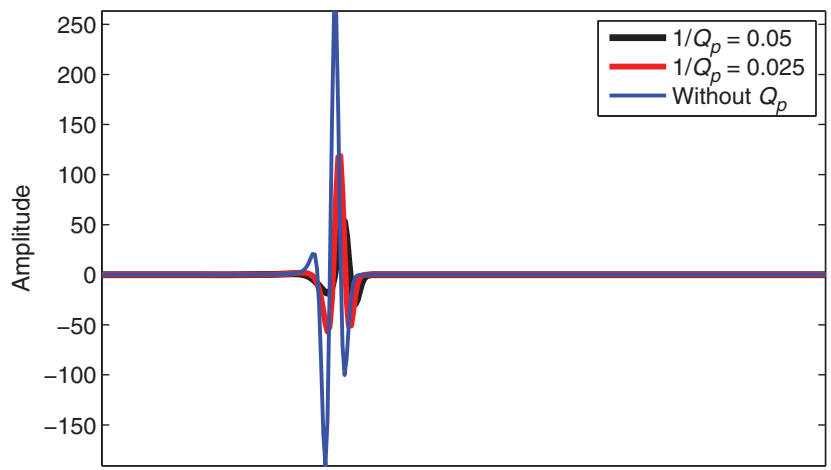

c)

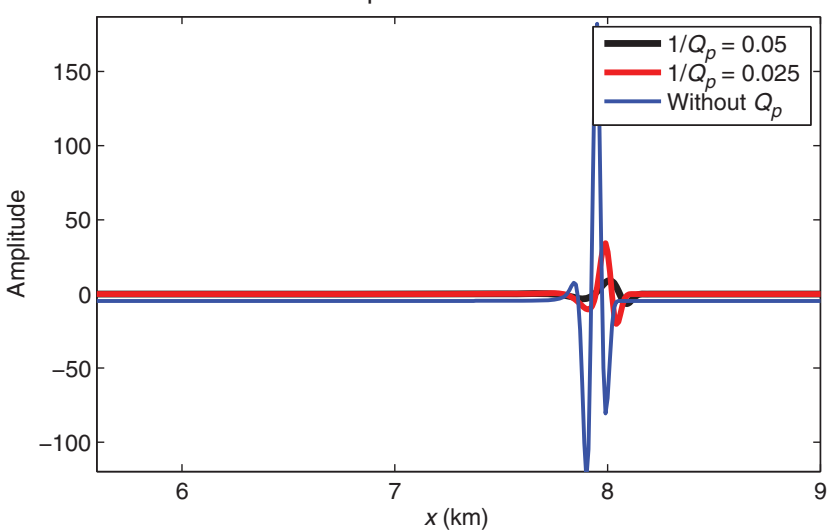

Figure 2. Depth slices from Figure 1 showing the effect of attenuation on the amplitude and phase of a propagating wave.

$$
\begin{aligned}
& \rho=\rho_{0}+\delta \rho, \\
& K=K_{0}+\delta K, \\
& \tau_{\sigma}=\tau_{\sigma_{0}}+\delta \tau_{\sigma}, \\
& \tau=\tau_{0}+\delta \tau .
\end{aligned}
$$

The perturbed wavefields can thus be written as

$$
\begin{aligned}
& \frac{\partial \delta P}{\partial t}+K \delta \tau(\nabla \cdot \mathbf{v})+K(\tau+1)(\nabla \cdot \delta \mathbf{v})+\delta r_{p} \\
& =-\delta K(\tau+1)(\nabla \cdot \mathbf{v}), \\
& \frac{\partial \delta \mathbf{v}}{\partial t}+\frac{1}{\rho} \nabla \delta P=\frac{\delta \rho}{\rho^{2}} \nabla P, \\
& \frac{\partial \delta r_{p}}{\partial t}+\frac{1}{\tau_{\sigma}}\left(\delta r_{p}+\tau K(\nabla \cdot \delta \mathbf{v})\right) \\
& =-\frac{1}{\tau_{\sigma}}(K \delta \tau+\tau \delta K)(\nabla \cdot \mathbf{v})+\frac{\delta \tau_{\sigma}}{\tau_{\sigma}^{2}}\left(r_{p}+\tau K(\nabla \cdot \mathbf{v})\right) .
\end{aligned}
$$

To simplify equation 14 , we make the following assumptions:

- $\quad$ The density is constant; i.e., $\delta \rho=0$.

- The material relaxation parameters are constant; i.e., $\delta \tau=0$ and $\delta \tau_{\sigma}=0$.

Equation 14 thus simplifies to

$$
\begin{aligned}
& \frac{\partial \delta P}{\partial t}+K(\tau+1)(\nabla \cdot \delta \mathbf{v})+\delta r_{p}=-\delta K(\tau+1)(\nabla \cdot \mathbf{v}), \\
& \frac{\partial \delta \mathbf{v}}{\partial t}+\frac{1}{\rho} \nabla \delta P=0, \\
& \frac{\partial \delta r_{p}}{\partial t}+\frac{1}{\tau_{\sigma}}\left(\delta r_{p}+\tau K(\nabla \cdot \delta \mathbf{v})\right)=-\frac{\tau}{\tau_{\sigma}} \delta K(\nabla \cdot \mathbf{v}) .
\end{aligned}
$$

In the context of $Q_{p}$-LSRTM, equation 15 is equivalent to the matrix-vector operation $\mathbf{d}=\mathbf{L m}$. Here, $\mathbf{d}$ represents the Born-modeled data with attenuation and is given by the solution of the linearized equations in equation $15, \mathbf{L}$ is a linear modeling operator, and $\mathbf{m}$ is related to the bulk modulus of the medium.

The adjoint equations for equation 12 can be derived using the adjoint-state method (shown in Appendix A) and is given by (Blanch and Symes, 1995)

$$
\begin{aligned}
& \frac{\partial q}{\partial t}+\nabla \cdot\left(\frac{1}{\rho} \mathbf{u}\right)=-\Delta d\left(\mathbf{x}_{g}, t ; \mathbf{x}_{s}\right), \\
& \frac{\partial \mathbf{u}}{\partial t}+\left[\nabla K(1+\tau) q+\nabla\left(\frac{1}{\tau_{\sigma}} K \tau s\right)\right]=0, \\
& \frac{\partial s}{\partial t}-\frac{s}{\tau_{\sigma}}-q=0 .
\end{aligned}
$$

Here, $(q, \mathbf{u}, s)$ are the adjoint-state variables of the state variables $\left(P, \mathbf{v}, r_{p}\right)$ and $\Delta d\left(\mathbf{x}_{q}, t ; \mathbf{x}_{s}\right)$ represents the residual pressure seismogram. For $Q_{p}$-LSRTM, $\Delta d\left(\mathbf{x}_{g}, t ; \mathbf{x}_{s}\right)$ represents the data residual between the predicted and the observed pressure data at every iteration.

The perturbation in the image $\delta m$ is related to the perturbation in the bulk modulus $\delta K$, which in turn can be obtained by zero-lag 
crosscorrelation of the adjoint fields from equation 16 with the background wavefields from equation 12 as

$$
\delta m \approx \delta K=\int_{0}^{T}(1+\tau)(\nabla \cdot \mathbf{v}) q+\frac{\tau}{\tau_{\sigma}}(\nabla \cdot \mathbf{v}) s d t .
$$

The solution to equation 17 is equivalent to the $\mathbf{g}=\mathbf{L}^{T} \Delta \mathbf{d}$ step in $Q_{p}$-LSRTM. A detailed derivation of the adjoint equations and the gradient is shown in Appendix A.

The next subsection describes the algorithm for numerical implementation of $Q_{p}$-LSRTM.

\section{$Q_{p}$-least-squares reverse time migration algorithm}

The following steps are carried out for numerically implementing $Q_{p}$-LSRTM by a preconditioned conjugate gradient method, in which a diagonal preconditioning matrix $\mathbf{C}$ is assumed. In this work, source-side illumination (Plessix and Mulder, 2004) is used as the diagonal preconditioner:

- Form the misfit function $\epsilon$ as

$$
\epsilon=\frac{1}{2}\left\|\mathbf{L} \mathbf{m}^{(i+1)}-\mathbf{d}^{\mathrm{obs}}\right\|^{2},
$$

where $\mathbf{L}$ represents a linear modeling operator and $\mathbf{L} \mathbf{m}^{(i+1)}$ is the predicted data given by the solution to equation $15 ; \mathbf{d}^{\text {obs }}$ represents the recorded pressure seismogram, $\mathbf{m}$ represents the reflectivity image, and $i$ represents the iteration index.

- Compute the gradient given by

$$
\mathbf{g}^{(i+1)}=\mathbf{L}^{T}\left[\mathbf{L} \mathbf{m}^{(i+1)}-\mathbf{d}^{\text {obs }}\right]=\mathbf{L}^{T} \Delta \mathbf{d}^{(i+1)},
$$

where $\Delta \mathbf{d}$ represents the data residual for the predicted and observed data, which is back-propagated by using the adjoint equations in equation 16. The adjoint wavefields are crosscorrelated with the background fields, given in equation 9, to give the perturbation in bulk reflectivity in equation 17 at each iteration. This perturbation can then be suitably scaled to give the perturbation in the reflectivity image $\delta m$ as

$$
\begin{aligned}
& K=\rho v^{2} \Rightarrow \delta K=2 \rho v \delta v(\because \delta \rho=0) \\
& \therefore \delta m=\frac{\delta v}{v}=\frac{\delta K}{2 \rho v^{2}} .
\end{aligned}
$$

- Update the gradient using the conjugate gradient formula as

$$
\mathbf{d k}^{(i+1)}=\mathbf{C g}^{(i+1)}+\beta \mathbf{d} \mathbf{k}^{(i)},
$$

where $\beta$ is given by

$$
\beta=\frac{\left(\mathbf{g}^{(i+1)}\right)^{T} \mathbf{C} \mathbf{g}^{(i+1)}}{\left(\mathbf{g}^{(i)}\right)^{T} \mathbf{C} \mathbf{g}^{(i)}} .
$$

- $\quad$ Compute the step length $\alpha$ as

$$
\alpha=\frac{\left(\mathbf{d k}^{(i+1)}\right)^{T} \mathbf{g}^{(i+1)}}{\left(\mathbf{L} \mathbf{d} \mathbf{k}^{(i+1)}\right)^{T}\left(\mathbf{L} \mathbf{d} \mathbf{k}^{(i+1)}\right)} .
$$

- Iteratively update the reflectivity image as

$$
\mathbf{m}^{(i+2)}=\mathbf{m}^{(i+1)}+\alpha \mathbf{d} \mathbf{k}^{(i+1)},
$$

until the length of the residual vector falls below a specified threshold.

\section{NUMERICAL RESULTS}

The effectiveness of $Q_{p}$-LSRTM is now demonstrated with synthetic and field data records from a crosswell experiment in Friendswood, Texas. The synthetic examples are for two models with strong attenuation: (1) a layered model with a shallow velocity and $Q_{p}$ anomaly, and 2) the Marmousi model.

In the synthetic examples, the observed data are generated by an $O(2,8)$ time-space-domain staggered-grid solution of the viscoacoustic-wave equation in equation 9. A Ricker wavelet with a peak frequency of $20 \mathrm{~Hz}$ is chosen as the source wavelet. The data are then migrated by using RTM, LSRTM, $Q_{p}$-RTM, and $Q_{p}$-LSRTM. Here, RTM and $Q_{p}$-RTM refer to the first iteration of LSRTM and $Q_{p^{-}}$ LSRTM, respectively. Source-side illumination is used as the preconditioning factor during the least-squares iterations for LSRTM and $Q_{p}$-LSRTM. The standard RTM and $Q_{p}$-RTM images are also illumination compensated.

\section{Layered velocity model}

We first demonstrate the effect of strong subsurface attenuation on migrated images using a simple example of a flat layered model. Figure 3 shows a layered velocity model with a shallow $Q_{p}$ anomaly. The $Q_{p}$ value in the anomaly is 20 , implying very strong attenuation for a wave propagating through this layer. To generate the synthetic data, equation 9 is solved for 100 shots evenly spaced at $40 \mathrm{~m}$ on the surface. Here, 200 receivers are evenly distributed at $20 \mathrm{~m}$ on the surface.

Figure $4 \mathrm{a}$ and $4 \mathrm{~b}$ compares the RTM and LSRTM images when the data having strong attenuation are migrated under the acoustic approximation. The LSRTM image has fewer artifacts and better
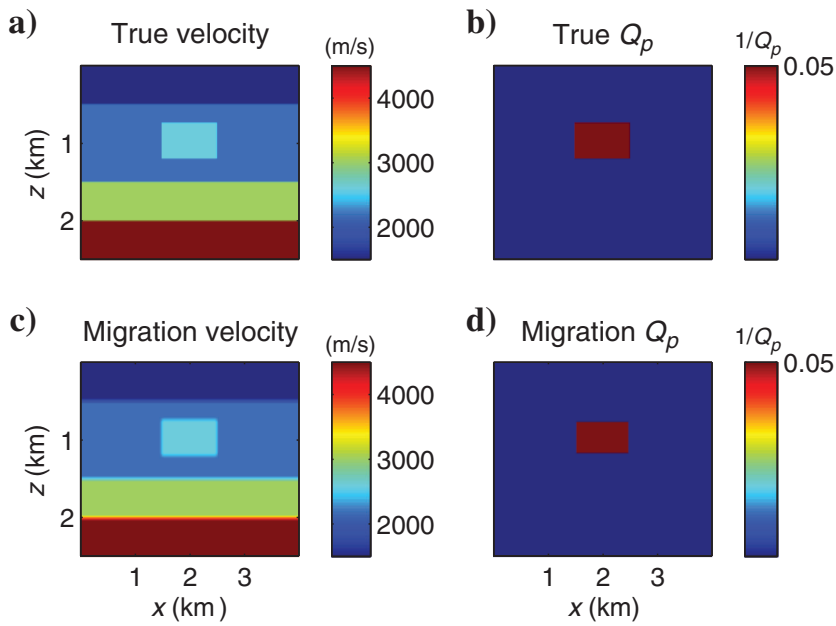

Figure 3. A layered model: (a) true velocity model (b) true $Q_{p}$ model (c) migration velocity model (d) $Q_{p}$ model used for $Q_{p^{-}}$ RTM and $Q_{p}$-LSRTM. 
balanced amplitudes in the shallow layers compared with the RTM image. However, the deeper layers below the anomaly (shown by the black arrows in Figure 4) have very weak amplitudes in the RTM and the LSRTM images. There is also a slight mispositioning in their locations. This is because strong attenuation affects the amplitudes and the phases of the propagating waves.

The $Q_{p}$-RTM and $Q_{p}$-LSRTM images after 20 iterations are shown in Figure $4 \mathrm{c}$ and $4 \mathrm{~d}$, respectively. The shallow $Q_{p}$-LSRTM image is similar to that for standard LSRTM in terms of artifact mitigation and better balancing of reflector amplitudes. However, improvements with $Q_{p}$-LSRTM are evident at the base of the anomaly and the reflectors directly beneath it (shown by the black arrows in Figure 4 and in the magnified views in Figure 5). The reflectors are imaged at the correct locations, and the migration amplitudes of these layers are more accurate than those obtained by standard LSRTM. As shown in Figure 5, $Q_{p}$-LSRTM has corrected for the
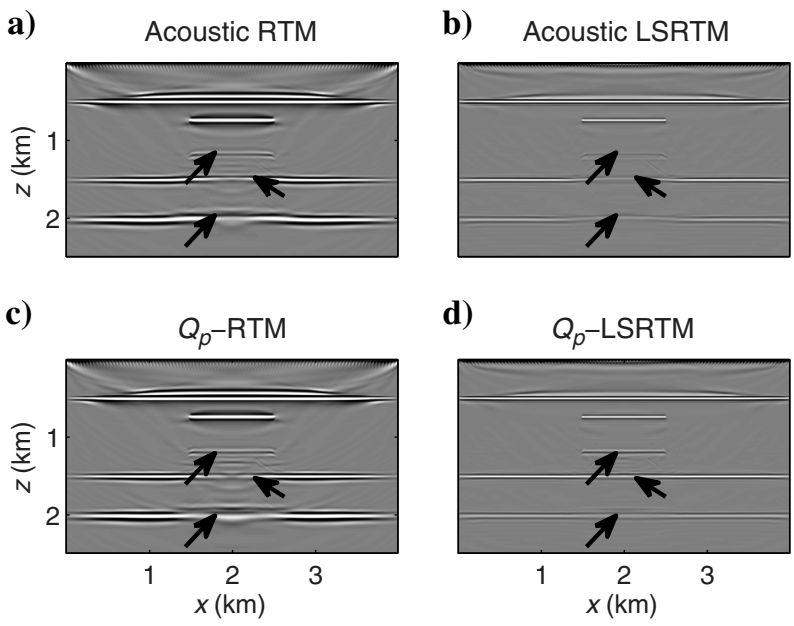

Figure 4. Comparison among images from (a) acoustic RTM, (b) acoustic LSRTM, (c) $Q_{p}$-RTM, and (d) $Q_{p}$-LSRTM. The black arrows point to the reflectors below the strong attenuation layer where improvements from $Q_{p}$-LSRTM can be seen. a)

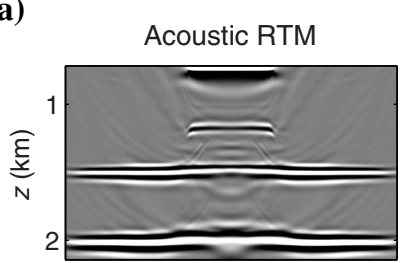

c)

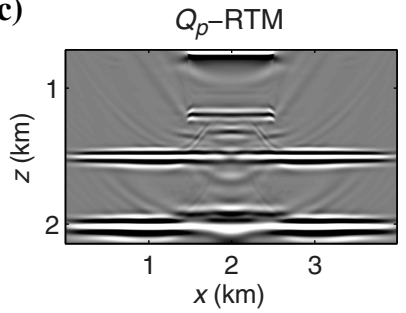

b)

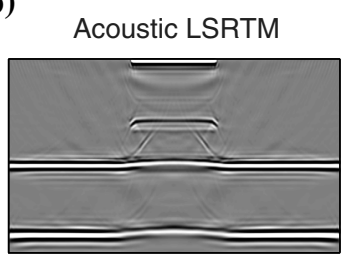

d)

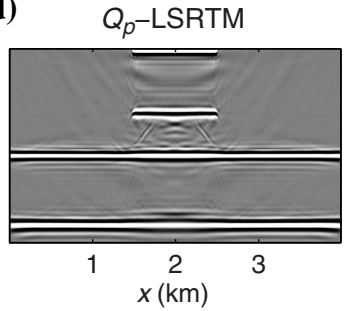

Figure 5. Magnified views of Figure 4 in which all the images have been normalized such that the short reflector at the top of the $Q_{p}$ anomaly have the same magnitude. amplitude loss and the phase distortion in the deeper layers due to the overlying $Q_{p}$ anomaly.

\section{Marmousi model}

The $Q_{p}$-LSRTM method is now tested on the more complex Marmousi model. Figure $6 \mathrm{a}$ and $6 \mathrm{~b}$ shows the true velocity and $Q_{p}$ models, respectively, used for generating the observed data. The migration velocity and $Q_{p}$ models are shown in Figure 6c and 6d, respectively, and the $Q_{p}$ model is chosen such that the attenuation layers are overlying the targeted deeper anticlines. The observed synthetic data are generated with a fixed spread geometry in which 200 shots are excited with a 40-m shot interval at a depth of $10 \mathrm{~m}$. Each shot is recorded with 400 receivers and a $20-\mathrm{m}$ receiver interval with a recording time of approximately $8 \mathrm{~s}$.

Conventional acoustic RTM and LSRTM images are displayed in Figure $7 \mathrm{a}$ and $7 \mathrm{~b}$, respectively. Similar to the previous example, the LSRTM image shows better resolution and fewer artifacts in the a)
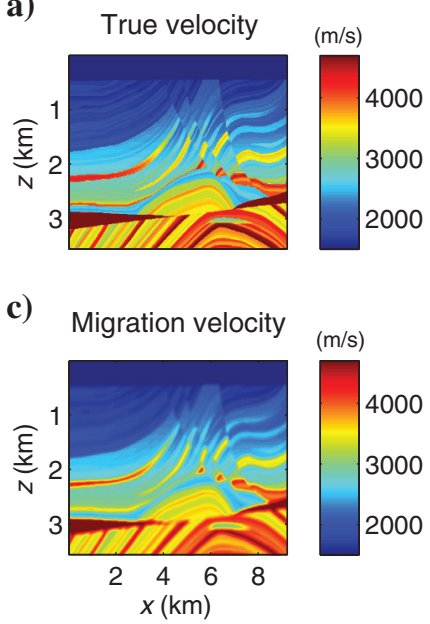

b)

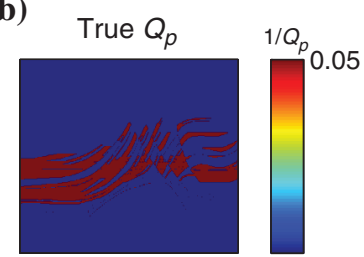

d)

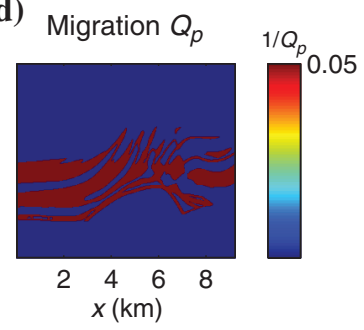

Figure 6. The modified Marmousi models: (a) true velocity model, (b) true $Q_{p}$ model, (c) migration velocity model, and (d) $Q_{p}$ model used for $Q_{p}$-RTM and $Q_{p}$-LSRTM.
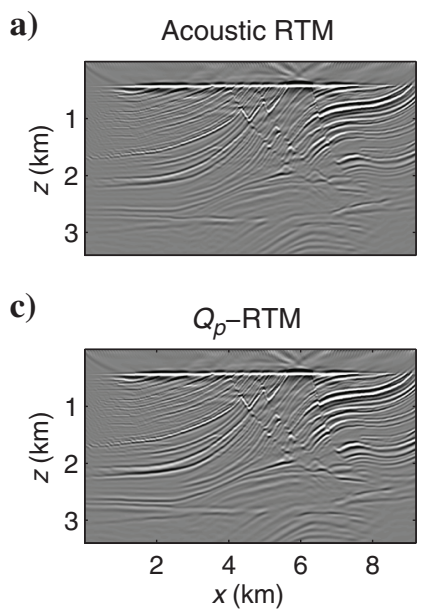

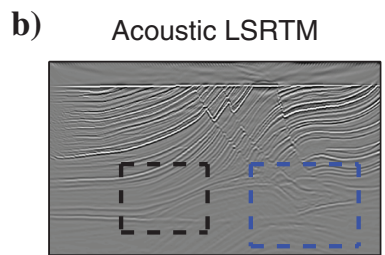

d)

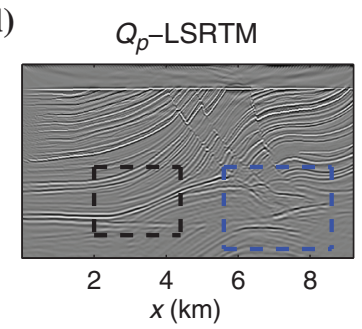

Figure 7. Comparison among images from (a) acoustic RTM, (b) acoustic LSRTM, (c) $Q_{p}$-RTM, and (d) $Q_{p}$-LSRTM. The black and blue boxes point to the areas for the magnified views. 
shallow layers compared with the standard RTM image. However, in the deeper layers, the amplitudes of the images from these two imaging methods are very weak. The reflectors and the anticlines cannot be properly delineated in spite of using a very accurate velocity model for migration. This distortion can be attributed to the strong attenuation above these layers.

The $Q_{p}$-RTM image, shown in Figure 7c, shows no improvements in the deeper layers compared to the standard RTM image in Figure 7a. However, significant improvements can be seen in the $Q_{p^{-}}$ LSRTM image shown in Figure 7d. In the shallow layers, the acoustic LSRTM and $Q_{p}$-LSRTM images are very similar in terms of the image quality. However, the amplitudes of the deeper reflectors and the anticlines are significantly better balanced in the $Q_{p}$-LSRTM image than in the standard RTM and LSRTM images. The magnified views of the black and blue boxes in Figure $7 \mathrm{~b}$ and $7 \mathrm{~d}$ are shown in Figure 8. The black arrows point to the areas in which noticeable improvements in the image quality can be seen with $Q_{p}$-LSRTM.

The residual as a function of iteration number for acoustic and $Q_{p}$-LSRTM is plotted in Figure 9. The convergence rate for $Q_{p^{-}}$ LSRTM is better than that for acoustic LSRTM because the correct physics of attenuation is accounted for in the forward and adjoint operators.

\section{Sensitivity of $Q_{p}$-least-squares reverse time migration to errors in the velocity model}

The sensitivity of $Q_{p}$-LSRTM to errors in the migration velocity model is now tested. For these numerical simulations, we assume that an accurate estimate of $Q_{p}$ is available. The errors are introduced into the migration velocity model by applying a triangle smoothing filter with increasing window lengths to the true velocity model in Figure 6a. The $Q_{p}$-LSRTM images for the different veloc-
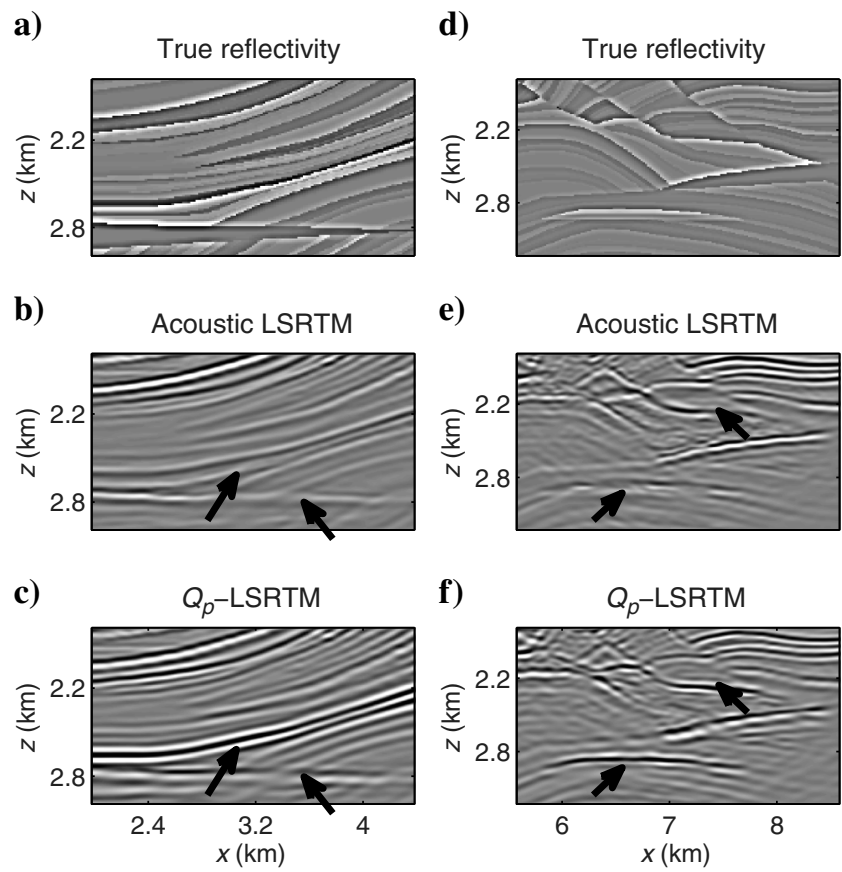

Figure 8. Magnified views of the black (left) and blue (right) boxes in Figure $7 \mathrm{~b}$ and $7 \mathrm{~d}$. (a and d) True reflectivity models used only for comparison, (b and e) acoustic LSRTM images, and (c and f) $Q_{p^{-}}$ LSRTM images. ity models are shown in Figure 10b. It becomes evident from these figures that the blurring in the $Q_{p}$-LSRTM image increases with depth for velocity errors exceeding approximately $8 \%$. The convergence curves, shown in Figure 11, show that the convergence of $Q_{p^{-}}$ LSRTM decreases with increasing errors in the velocity model.

\section{Sensitivity of $Q_{p}$-least-squares reverse time migration to errors in the $Q_{p}$ model}

To quantify the sensitivity of $Q_{p}$-LSRTM to errors in the $Q_{p}$ migration model, the $Q_{p}$-LSRTM images for different attenuation

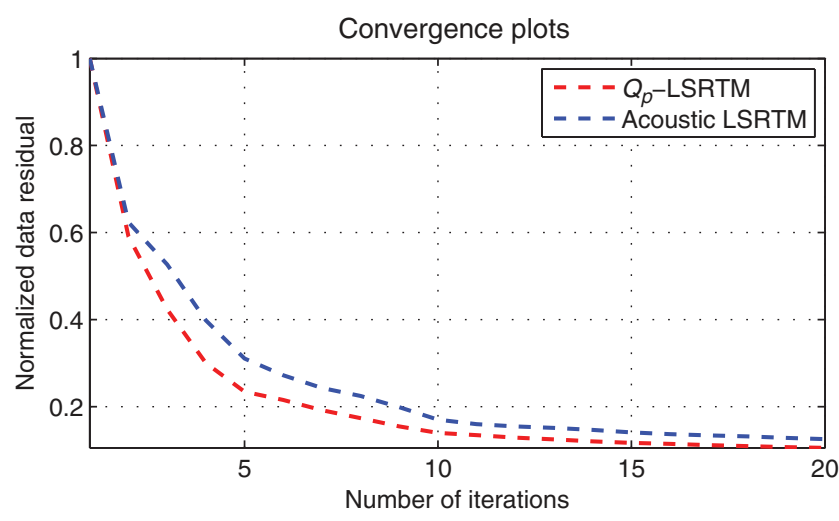

Figure 9. Convergence curves for acoustic and $Q_{p}$-LSRTM for the Marmousi model.
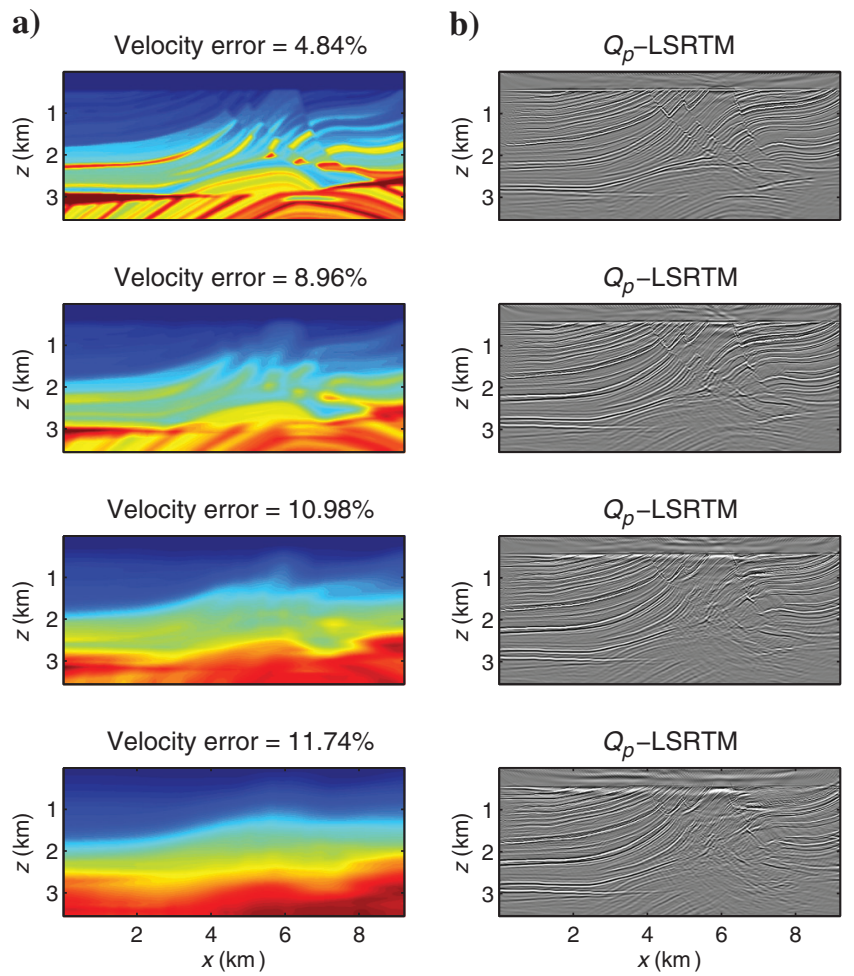

Figure 10. Sensitivity of $Q_{p}$-LSRTM to errors in the migration velocity model. (a) The figures in the left column show the different velocity models used for $Q_{p}$-LSRTM. (b) The figures in the right column are the corresponding $Q_{p}$-LSRTM images after 20 iterations. 
models are shown in Figure 12b. It is evident from these figures that $Q_{p}$-LSRTM shows improvement over standard LSRTM when the error in the migration $Q_{p}$ model is approximately $50 \%$. For attenuation errors exceeding $50 \%$, the amplitude loss is not compensated for by $Q_{p}$-LSRTM, and the inverted images are very similar to the acoustic LSRTM image shown in Figure $7 \mathrm{~b}$. The convergence curves for the different cases, shown in Figure 13, suggest that $Q_{p^{-}}$ LSRTM converges quickly even when there are significant errors in the migration $Q_{p}$ model. This is contrary to the convergence curves shown in Figure 11 in which the convergence of $Q_{p}$-LSRTM

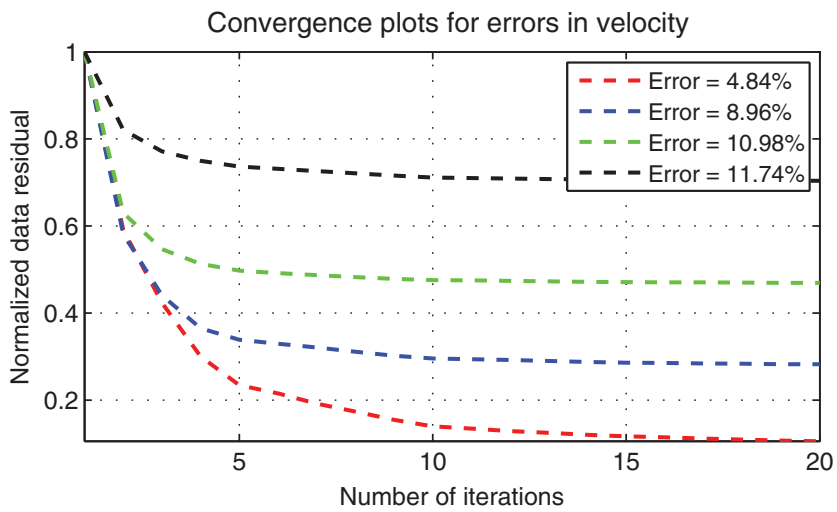

Figure 11. Normalized data residual versus iteration number for $Q_{p}$-LSRTM with different percentage errors in the migration velocity model. a)

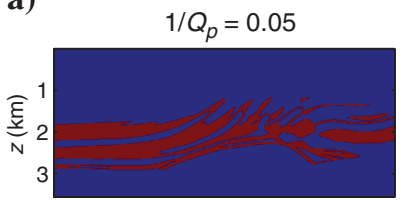

$1 / Q_{p}=0.025$
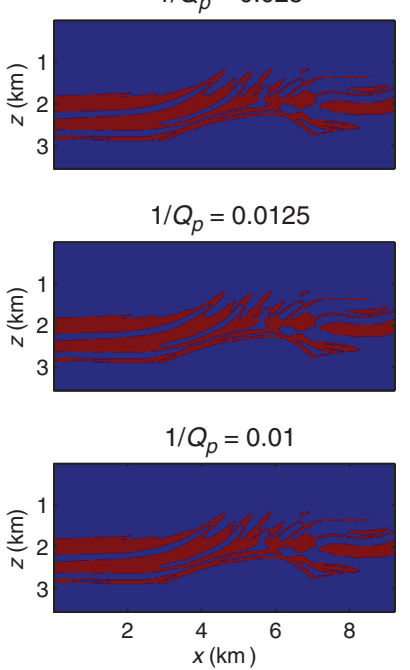

b)
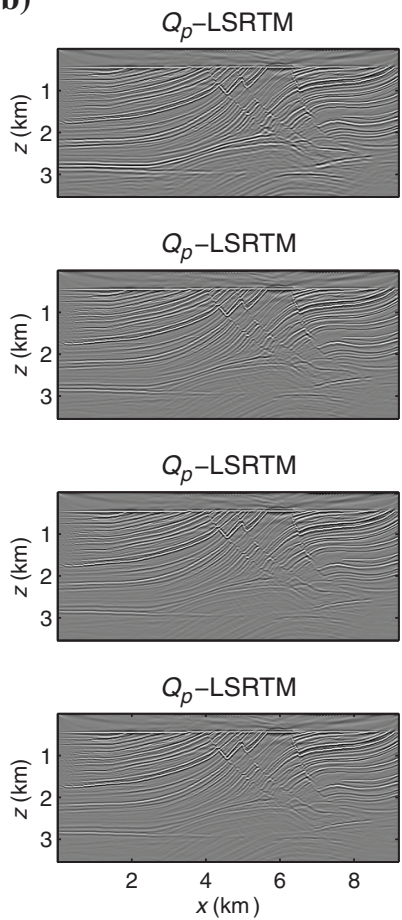

Figure 12. Sensitivity of $Q_{p}$-LSRTM to errors in the migration $Q_{p}$ model. (a) The figures in the left column show the different $Q_{p}$ models used for $Q_{p}$-LSRTM. The true model had a background $Q_{p}$ value of 0.05 . (b) The figures in the right column are the corresponding $Q_{p}$-LSRTM images after 20 iterations for different background $Q_{p}$ values. becomes slower with increasing errors in the migration velocity model. These curves suggest that the convergence rate of $Q_{p^{-}}$ LSRTM is more sensitive to errors in the velocity model than to errors in the $Q_{p}$ model. However, with increasing errors in the migration $Q_{p}$ model, the amplitude loss due to attenuation is not corrected for by $Q_{p}$-LSRTM. Thus, a fairly accurate estimation of the $Q_{p}$ distribution is required to see noticeable improvements in the image quality with $Q_{p}$-LSRTM.

\section{Friendswood crosswell field data}

As a final example, $Q_{p}$-LSRTM is applied to the Friendswood crosswell data (Chen et al., 1990). Two 305-m-deep cased wells separated by $183 \mathrm{~m}$ were used as the source and receiver wells. Downhole explosive sources of 10-g charges were fired at intervals of $3 \mathrm{~m}$ from 305 to $9 \mathrm{~m}$ in the source well and the receiver well had 96 receivers placed at depths ranging from 293 to $3 \mathrm{~m}$. The data were recorded with a sampling interval of $0.25 \mathrm{~ms}$ for a total recording time of $0.375 \mathrm{~s}$. The following processing steps were first applied to the data:

- The recorded data are corrected from $3 \mathrm{D}$ to $2 \mathrm{D}$ format by scaling the amplitudes by $\sqrt{t}$ to approximate geometrical spreading. A phase correction is applied by multiplying the spectrum of the observed seismogram with the filter $\sqrt{i / \omega}$ (Zhou et al., 1995).

- A directional nine-point median filter is used to eliminate the tube waves that are seen as linear events in the common shot gather (CSG) shown in Figure 14a. To implement this filter, a linear moveout correction is applied to the CSG to flatten the tube wave arrivals so that they arrive at the same time. For every time sample, a nine-trace median filter is applied to the traces so that the output traces have highly amplified tube waves and diminished reflection events around the onset of the tube waves. These predicted tube waves are then subtracted from the original data.

- A band-pass filter of $80-600 \mathrm{~Hz}$ is applied to the data to remove any extreme noise from the data. The final processed CSG after applying all these processing steps is shown in Figure 14b.

- Because the bandwidth of $80-600 \mathrm{~Hz}$ is too broad for waveform tomography and $Q_{p}$-LSRTM, the data are Wiener-

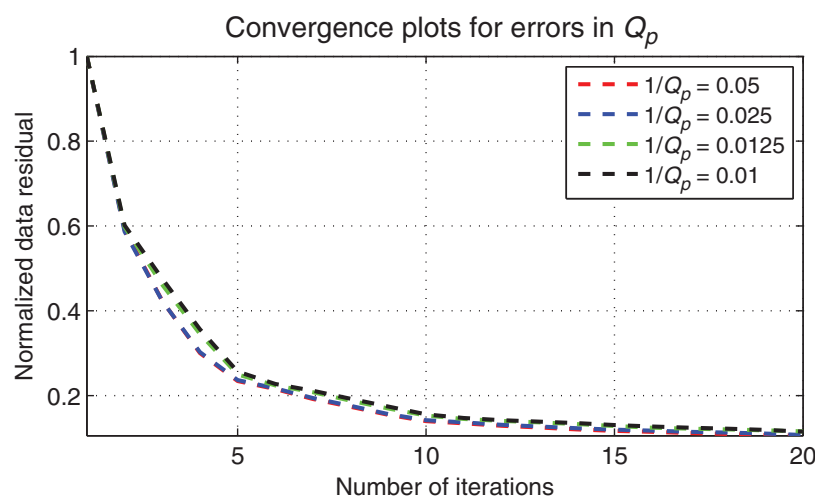

Figure 13. Normalized data residual versus iteration number for $Q_{p}$-LSRTM with different migration $Q_{p}$ models. 
filtered to transform the original wavelet to a Ricker wavelet with a $200-\mathrm{Hz}$ peak frequency. This bandwidth is chosen based on the frequency content of the data in which most of the signal is concentrated between 150 and $250 \mathrm{~Hz}$.

- The first-arrival traveltimes are then picked from all the CSGs and inverted to get a starting velocity model for early arrival waveform inversion. The early arrival waveform tomogram, shown in Figure 15a, is used as the migration velocity model. The attenuation tomography method of Quan and Harris (1997) is used to get a reference $Q_{p}$ model for $Q_{p}$-RTM and $Q_{p}$-LSRTM. The estimated $Q_{p}$ tomogram is shown in Figure 15b.

The images from acoustic LSRTM and $Q_{p}$-LSRTM after 30 iterations are shown in Figure 16b and 16d, respectively. The decrease in residual was approximately $25 \%-30 \%$ for both inversions. In the shallow part of the image between 25 and $120 \mathrm{~m}$, the amplitudes of the reflectors have improved in the $Q_{p}$-LSRTM image compared with the acoustic LSRTM image. In the deeper part between 170 and $200 \mathrm{~m}$, the layers have been better delineated in the $Q_{p}$-LSRTM image. The lateral continuity of the imaged reflectors has also improved in this area. Magnified views of the black boxes in Figure 16, shown in Figure 17, further emphasize the improvement in the image quality from $Q_{p}$-LSRTM.
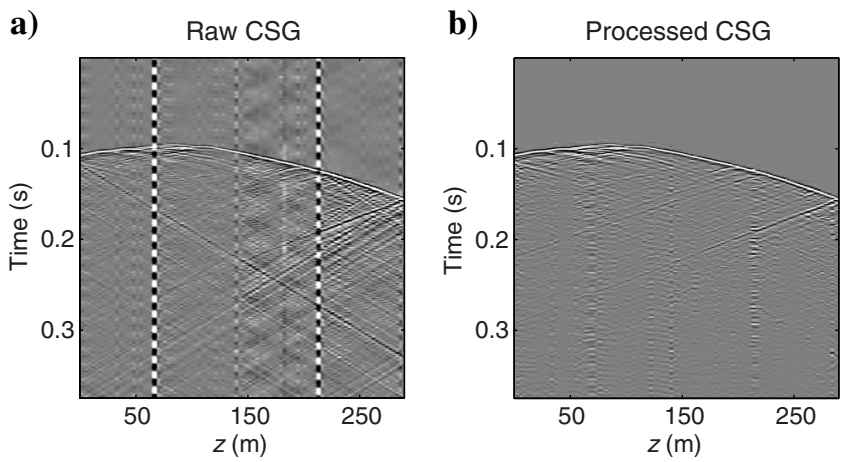

Figure 14. (a) A raw CSG from the Friendswood crosswell data. (b) The same CSG after applying all the data-processing steps.
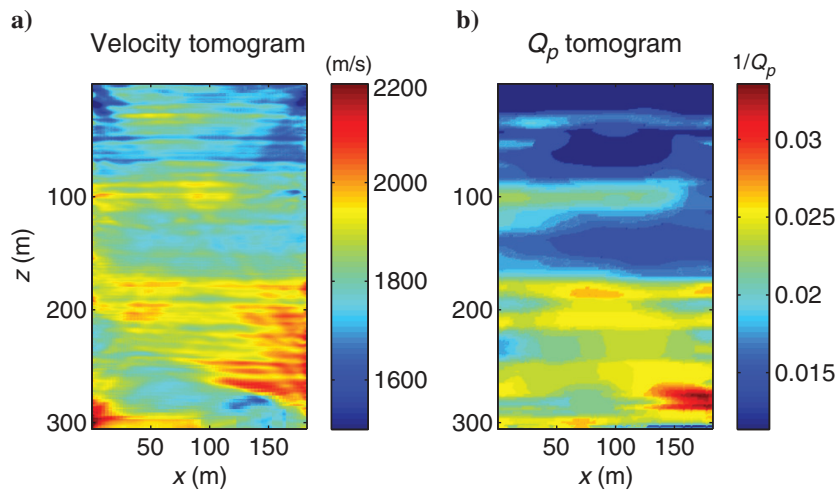

Figure 15. (a) Velocity tomogram estimated for the crosswell data using early arrival waveform inversion. (b) The $Q_{p}$-tomogram estimated using attenuation tomography.

\section{DISCUSSIONS}

The estimation of $Q_{p}$ is often ambiguous and inaccurate. Though several methods for estimating $Q_{p}$ from surface seismic data have been proposed in the past by Brzostowski and McMechan (1992), Hicks and Pratt (2001), Cavalca and Fletcher (2008), Bai et al. (2012), and Valenciano and Chemingui (2013), getting a reliable background $Q_{p}$ model for least-squares migration or full-waveform a)

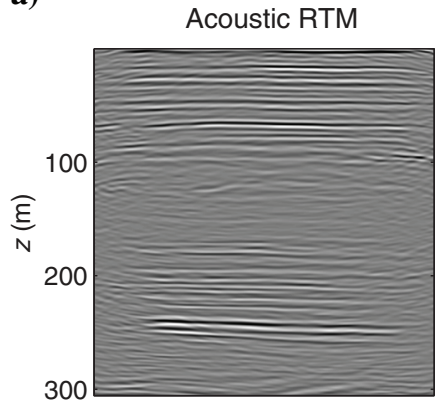

c)

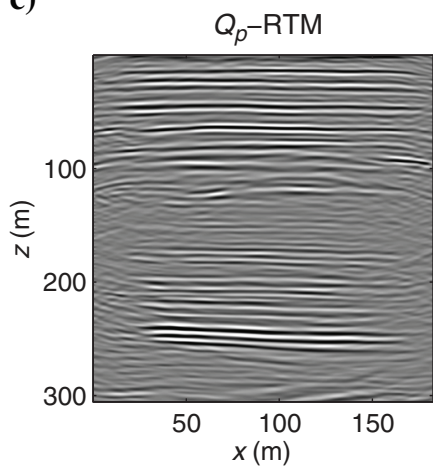

b)

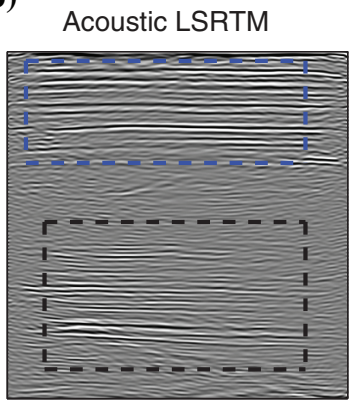

d)

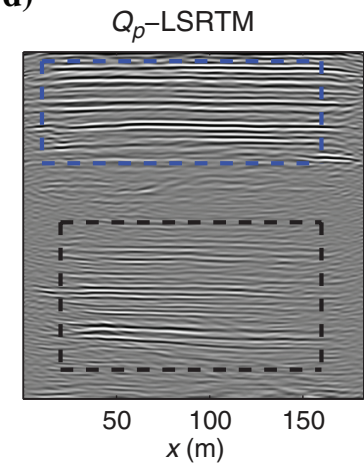

Figure 16. Comparison among images from (a) acoustic RTM, (b) acoustic LSRTM, (c) $Q_{p}$-RTM, and (d) $Q_{p}$-LSRTM after 30 iterations. The black boxes point to the areas for the magnified views. a)

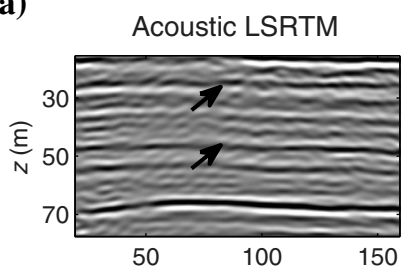

c) Acoustic LSRTM

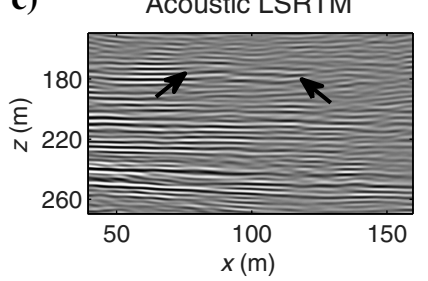

b)

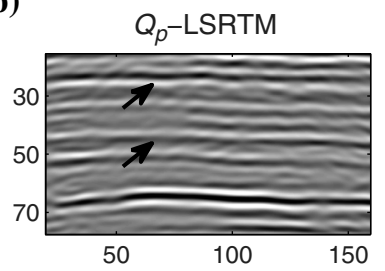

d)

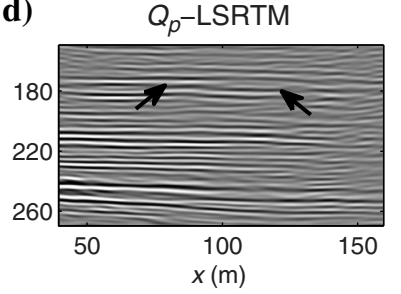

Figure 17. Magnified views of the black boxes in Figure 16b and $16 \mathrm{~d}$. The black areas point to the areas in which improvements can be seen. 
inversion is still difficult compared with estimating the background velocity model. Our sensitivity tests for the background velocity and $Q_{p}$ have shown that the quality of the inverted image from $Q_{p}$-LSRTM is sensitive to both of them. For our field data tests, we used the $Q_{p}$ tomogram, shown in Figure 15b, as a reference and varied the background $Q_{p}$ until reasonable improvements in the image quality could be seen from $Q_{p}$-LSRTM. If the background $Q_{p}$ is increased too much, the results became similar to the acoustic case. If the background $Q_{p}$ is made smaller, the high-frequency content in the $Q_{p}$-LSRTM image got damped down because the least-squares inversion compensated for the wrong $Q_{p}$ model by damping down the high wavenumbers in the image. The results shown in Figure 16 are obtained by scaling the $Q_{p}$ tomogram in Figure 15 by $50 \%$.

Throughout our numerical tests, we assumed a single relaxation mechanism, which is valid if the bandwidth of the data is narrow. For a wide bandwidth of the data, as in the case of the Friendswood crosswell data, a single relaxation mechanism may not be sufficient to accurately model the effect of $Q_{p}$. However, using more than one relaxation mechanism will significantly increase the computational cost of $Q_{p}$-LSRTM. For typical exploration problems for RTM in which the bandwidth of the data used is approximately $5-30 \mathrm{~Hz}$, use of a single relaxation mechanism should suffice (Blanch et al., 1995). Also, in our numerical simulations, we have assumed that the recorded data are free from $\mathrm{S}$ waves. Similar to standard LSRTM, if there are shear waves present in the data, $Q_{p}$-LSRTM will produce images with strong $\mathrm{S}$-wave artifacts.

A disadvantage of $Q_{p}$-LSRTM is that the computational cost per iteration is more than six times that of standard RTM. The cost increases linearly with the number of least-squares iterations. As in the case of standard least-squares migration, $Q_{p}$-LSRTM can be made more efficient using multisource-encoded migration (Dai and Schuster, 2009; Tang, 2009; Dai et al., 2010; Huang and Schuster, 2012) where several shot gathers are randomly shifted in time to form a phase-encoded supergather.

\section{CONCLUSIONS}

A time-domain LSRTM method is presented that uses the viscoacoustic-wave equation to compensate for the distortion in amplitudes and phases of seismic waves propagating in highly attenuative layers. Numerical results on synthetic and field data validate that if the recorded data have strong attenuation, then conventional acoustic RTM and LSRTM cannot correct for the attenuation loss. However, if the linearized viscoacoustic-wave equation and its adjoint equations are used for LSRTM, the attenuation loss can be compensated during the iterations. Results with synthetic and field data for strongly attenuative media show that LSRTM with $Q_{p}$ compensation produces images with better balanced amplitudes and accurately positioned reflectors compared with acoustic RTM and LSRTM. Similar to standard LSRTM, $Q_{p}$-LSRTM is also sensitive to errors in the migration velocity model. Another input requirement for this method is an accurate estimate of the smoothly varying $Q_{p}$ distribution in the subsurface. Accurate estimation of $Q_{p}$ coupled with the linearized $Q_{p}$-LSRTM method proposed in this paper has the potential for accurate imaging in highly attenuative geologic environments such as gas-sandstones and shales.

\section{ACKNOWLEDGMENTS}

The research reported in this publication was supported by the King Abdullah University of Science and Technology (KAUST) in Thuwal, Saudi Arabia. We are grateful to the sponsors of the Center for Subsurface Imaging and Modeling (CSIM) Consortium for their financial support. The first author would also like to thank W. Dai for his help in viscoacoustic modeling. For computer time, this research used the resources of the Supercomputing Laboratory at KAUST. We thank them for providing the computational resources required for carrying out this work. We also thank the associate editor J. Behura and three anonymous reviewers whose meticulous reviews have greatly improved the quality of this manuscript.

\section{APPENDIX A}

\section{ADJOINT EQUATIONS AND GRADIENTS FOR $Q_{P^{-}}$ LEAST-SQUARES REVERSE TIME MIGRATION}

In matrix-vector notation, equation 12 can be rewritten as

$$
\left[\begin{array}{ccc}
\frac{\partial}{\partial t} & K(1+1) \nabla \cdot & 1 \\
\frac{1}{\rho} \nabla & \frac{\partial}{\partial t} & 0 \\
0 & \frac{\tau}{\tau_{\sigma}} K \nabla \cdot & \frac{\partial}{\partial t}+\frac{1}{\tau_{\sigma}}
\end{array}\right]\left[\begin{array}{c}
P \\
\mathbf{v} \\
r_{p}
\end{array}\right]=\left[\begin{array}{l}
f \\
0 \\
0
\end{array}\right],
$$

or in a more compact form as

$$
\mathbf{S w}=\mathbf{F} .
$$

where

$\mathbf{S}=\left[\begin{array}{ccc}\frac{\partial}{\partial t} & K(1+1) \nabla . & 1 \\ \frac{1}{\rho} \nabla & \frac{\partial}{\partial t} & 0 \\ 0 & \frac{\tau}{\tau_{\sigma}} K \nabla \cdot & \frac{\partial}{\partial t}+\frac{1}{\tau_{\sigma}}\end{array}\right], \quad \mathbf{w}=\left[\begin{array}{c}P \\ \mathbf{v} \\ r_{p}\end{array}\right]$, and $\mathbf{F}=\left[\begin{array}{c}f \\ 0 \\ 0\end{array}\right]$

Here, w represents the state variables and $\mathbf{S}$ represents the forward modeling operator. The adjoint operator $\mathbf{S}^{*}$ of $\mathbf{S}$ in this particular case is given by

$$
\mathbf{S}^{*}=\left[\begin{array}{ccc}
-\frac{\partial}{\partial t} & -\nabla \cdot \frac{1}{\rho} & 0 \\
-\nabla K(1+1) & -\frac{\partial}{\partial t} & -\nabla \frac{\tau}{\tau_{\sigma}} K \\
1 & 0 & -\frac{\partial}{\partial t}+\frac{1}{\tau_{\sigma}}
\end{array}\right] .
$$

The least-squares misfit functional $J(\mathbf{m})$ for a model parameter $\mathbf{m}$ can be written as

$$
J(\mathbf{m})=\frac{1}{2}\|\mathbf{w}(\mathbf{m})-\mathbf{d}\|^{2}=\frac{1}{2}\langle\mathbf{w}(\mathbf{m})-\mathbf{d}, \mathbf{w}(\mathbf{m})-\mathbf{d}\rangle,
$$

where $\mathbf{w}(\mathbf{m})$ and $\mathbf{d}$ represent the modeled and recorded data vectors, respectively. For a viscoacoustic medium, the model parameter $\mathbf{m}$ can be $K, \rho$, or $\tau$. The gradient of $J$ is given by 


$$
\frac{\partial J(\mathbf{m})}{\partial \mathbf{m}}=\left\langle\frac{\partial \mathbf{w}(\mathbf{m})}{\partial \mathbf{m}}, \mathbf{w}(\mathbf{m})-\mathbf{d}\right\rangle .
$$

Now considering a system of equations:

$$
\begin{aligned}
& S(\mathbf{m}) \mathbf{w}(\mathbf{m})=\mathbf{F}, \\
\Rightarrow & \frac{\partial S(\mathbf{m})}{\partial \mathbf{m}} \mathbf{w}(\mathbf{m})+S(\mathbf{m}) \frac{\partial \mathbf{w}(\mathbf{m})}{\partial \mathbf{m}}=0, \\
\Rightarrow & \frac{\partial \mathbf{w}(\mathbf{m})}{\partial \mathbf{m}}=-S^{-1}(\mathbf{m}) \frac{\partial S(\mathbf{m})}{\partial \mathbf{m}} \mathbf{w}(\mathbf{m}) .
\end{aligned}
$$

Inserting equation A-7 into equation A-6, we get

$$
\begin{aligned}
\frac{\partial J(\mathbf{m})}{\partial \mathbf{m}} & =-\left\langle S^{-1}(\mathbf{m}) \frac{\partial S(\mathbf{m})}{\partial \mathbf{m}} \mathbf{w}(\mathbf{m}), \mathbf{w}(\mathbf{m})-\mathbf{d}\right\rangle \\
& =-\left\langle\frac{\partial S(\mathbf{m})}{\partial \mathbf{m}} \mathbf{w}(\mathbf{m}),\left(S(\mathbf{m})^{-1}\right)^{*}(\mathbf{w}(\mathbf{m})-\mathbf{d})\right\rangle \\
& =-\left\langle\frac{\partial S(\mathbf{m})}{\partial \mathbf{m}} \mathbf{w}(\mathbf{m}),\left(S(\mathbf{m})^{-1}\right)^{*} \Delta \mathbf{d}\right\rangle \\
& =-\left\langle\frac{\partial S(\mathbf{m})}{\partial \mathbf{m}} \mathbf{w}(\mathbf{m}), \mathbf{w}^{*}(\mathbf{m})\right\rangle
\end{aligned}
$$

where * denotes the adjoint, $\Delta \mathbf{d}$ denotes the residual data vector, and $\mathbf{w}^{*}$ denotes the adjoint or the residual wavefield, which is obtained by solving the adjoint equations as

$$
S^{*}(\mathbf{m}) \mathbf{w}^{*}(\mathbf{m})=\Delta \mathbf{d} .
$$

$\mathbf{w}^{*}$ is also known as the adjoint state variable of $\mathbf{w}$. Let $\mathbf{w}^{*}$ be denoted as

$$
\mathbf{w}^{*}=[q \mathbf{u} s]^{T}
$$

where $q$ is the adjoint state variable of the pressure wavefield $P, \mathbf{u}$ is the adjoint of the particle velocity vector $\mathbf{v}$, and $s$ is the adjoint of the memory variable $r_{p}$. Assuming that we only record pressure seismograms, the residual vector $\Delta \mathbf{d}$ will have only one component, i.e.,

$$
\Delta \mathbf{d}=\left[\begin{array}{lll}
\Delta d & 0 & 0
\end{array}\right]^{T} .
$$

Using equations A-4, A-9, A-10, and A-11, we get the adjoint-state equations for a viscoacoustic medium as

$$
\begin{aligned}
& {\left[\begin{array}{ccc}
-\frac{\partial}{\partial t} & -\nabla \cdot \frac{1}{\rho} & 0 \\
-\nabla K(1+1) & -\frac{\partial}{\partial t} & -\nabla \frac{\tau}{\tau_{\sigma}} K \\
1 & 0 & -\frac{\partial}{\partial t}+\frac{1}{\tau_{\sigma}}
\end{array}\right]\left[\begin{array}{c}
q \\
\mathbf{u} \\
s
\end{array}\right]=\left[\begin{array}{c}
\Delta d \\
0 \\
0
\end{array}\right]} \\
& \frac{\partial q}{\partial t}+\nabla \cdot\left(\frac{1}{\rho} \mathbf{u}\right)=-\Delta d\left(\mathbf{x}_{g}, t ; \mathbf{x}_{s}\right) \\
& \frac{\partial \mathbf{u}}{\partial t}+\left[\nabla K(1+\tau) q+\nabla\left(\frac{1}{\tau_{\sigma}} K \tau s\right)\right]=0 \\
& \frac{\partial s}{\partial t}-\frac{s}{\tau_{\sigma}}-q=0 .
\end{aligned}
$$

For $\mathbf{m}=K$, the gradient in equation $\mathrm{A}-8$ can be written as

$$
\begin{aligned}
\frac{\partial J}{\partial K} & =-\left\langle\frac{\partial S}{\partial K} \mathbf{w}, \mathbf{w}^{*}\right\rangle \\
& =-\left\langle\left[\begin{array}{ccc}
0 & (1+\tau) \nabla \cdot & 0 \\
0 & 0 & 0 \\
0 & \frac{\tau}{\tau_{\sigma}} \nabla \cdot & 0
\end{array}\right]\left[\begin{array}{c}
P \\
\mathbf{v} \\
r_{p}
\end{array}\right],\left[\begin{array}{l}
q \\
\mathbf{u} \\
s
\end{array}\right]\right\rangle \\
& =-\int_{0}^{T}(1+\tau)(\nabla \cdot \mathbf{v}) q+\frac{\tau}{\tau_{\sigma}}(\nabla \cdot \mathbf{v}) s \mathrm{~d} t .
\end{aligned}
$$

\section{REFERENCES}

Aki, K., and P. G. Richards, 1980, Quantitative seismology: Freeman.

Bai, J., G. Chen, D. Yingst, and J. Leveille, 2013, Attenuation compensation in viscoacoustic reverse time migration: 83rd Annual International Meeting, SEG, Expanded Abstracts, 3825-3830.

Bai, J., D. Yingst, R. Bloor, and J. Leveille, 2012, Waveform inversion with attenuation: 82nd Annual International Meeting, SEG, Expanded Abstracts, doi: 10.1190/segam2012-1305.1.

Baysal, E., D. D. Kosloff, and J. W. Sherwood, 1983, Reverse time migration: Geophysics, 48, 1514-1524, doi: 10.1190/1.1441434.

Bickel, S., and R. Natarajan, 1985, Plane wave $Q$ deconvolution: Geophysics, 50, 1426-1439, doi: 10.1190/1.1442011.

Blanch, J., and W. Symes, 1995, Efficient iterative viscoacoustic linearized inversion: 65th Annual International Meeting, SEG, Expanded Abstracts, 627-630.

Blanch, J. O., J. O. Robertsson, and W. W. Symes, 1995, Modeling of a constant $Q$ : Methodology and algorithm for an efficient and optimally inexpensive viscoelastic technique: Geophysics, 60, 176-184, doi: 10 $.1190 / 1.1443744$.

Brzostowski, M. A., and G. A. McMechan, 1992, 3D tomographic imaging of near-surface seismic velocity and attenuation: Geophysics, 57, 396403, doi: 10.1190/1.1443254.

Carcione, J. M., D. Kosloff, and R. Kosloff, 1988, Wave propagation simulation in a linear viscoacoustic medium: Geophysical Journal International, 93, 393-401, doi: 10.1111/j.1365-246X.1988.tb02010.x.

Cavalca, M., and R. Fletcher, 2008, Deriving 3D $Q$ models from surface seismic data using attenuated traveltime tomography: 78th Annual Conference and Exhibition, EAGE, Extended Abstracts, H003.

Chen, S., L. Zimmerman, and J. Tugnait, 1990, Subsurface imaging using reversed vertical seismic profiling and crosshole tomographic methods: Geophysics, 55, 1478-1487, doi: 10.1190/1.1442795.

Christensen, R. M., 1982, Theory of viscoelasticity: An introduction: Academic Press.

Dai, N., and G. West, 1994, Inverse $Q$ migration: 64th Annual International Meeting, SEG, Expanded Abstracts, 1418-1421.

Dai, W., C. Boonyasiriwat, and G. T. Schuster, 2010, 3D multi-source leastsquares reverse time migration: 80th Annual International Meeting, SEG, Expanded Abstracts, 3120-3124.

Dai, W., P. Fowler, and G. T. Schuster, 2012, Multisource least-squares reverse time migration: Geophysical Prospecting, 60, 681-695, doi: 10 $.1111 / \mathrm{j} .1365-2478.2012 .01092 . x$.

Dai, W., and G. T. Schuster, 2009, Least-squares migration of simultaneous sources data with a deblurring filter: 79th Annual International Meeting, SEG, Expanded Abstracts, 2090-2994.

Dai, W., and G. T. Schuster, 2010, Multi-source wave equation least-squares migration with a deblurring filter: 80th Annual Conference and Exhibition, EAGE, Extended Abstracts, 276.

Dai, W., X. Wang, and G. T. Schuster, 2011, Least-squares migration of multisource data with a deblurring filter: Geophysics, 76, no. 5, R135-R146, doi: 10.1190/geo2010-0159.1.

Duquet, B., K. J. Marfurt, and J. A. Dellinger, 2000, Kirchhoff modeling, inversion for reflectivity, and subsurface illumination: Geophysics, 65 1195-1209, doi: 10.1190/1.1444812.

Fletcher, R., D. Nichols, and M. Cavalca, 2012, Wavepath-consistent effective $Q$ estimation for $Q$-compensated reverse time migration: 82nd Annual Conference and Exhibition, EAGE, Extended Abstracts, A020.

Hargreaves, N., and A. Calvert, 1991, Inverse $Q$ filtering by Fourier transform: Geophysics, 56, 519-527, doi: 10.1190/1.1443067.

Hicks, G. J., and R. G. Pratt, 2001, Reflection waveform inversion using local descent methods: Estimating attenuation and velocity over a gassand deposit: Geophysics, 66, 598-612, doi: 10.1190/1.1444951. 
Huang, Y., and G. T. Schuster, 2012, Multisource least-squares migration of marine streamer data with frequency-division encoding: Geophysical Prospecting, 60, 663-680, doi: 10.1111/j.1365-2478.2012.01086.x.

Kjartansson, E., 1979, Constant $Q$-wave propagation and attenuation: Journal of Geophysical Research, 84, 4737-4748, doi: 10.1029/ JB084iB09p04737.

Lailly, P., 1984, Migration methods: Partial but efficient solutions to the seismic inverse problem: Inverse Problems of Acoustic and Elastic Waves, 51, 1387-1403

McMechan, G., 1983, Migration by extrapolation of time-dependent boundary values: Geophysical Prospecting, 31, 413-420, doi: 10.1111/j.13652478.1983.tb01060.x.

Nemeth, T., C. Wu, and G. T. Schuster, 1999, Least-squares migration of incomplete reflection data: Geophysics, 64, 208-221, doi: 10.1190/1 .1444517.

Plessix, R.-E., 2006, A review of the adjoint-state method for computing the gradient of a functional with geophysical applications: Geophysical Journal International, 167, 495-503, doi: 10.1111/j.1365-246X.2006 .02978.x.

Plessix, R.-E., and W. A. Mulder, 2004, Frequency-domain finite-difference amplitude-preserving migration: Geophysical Journal International, 157, 975-987, doi: 10.1111/j.1365-246X.2004.02282.x.

Quan, Y., and J. M. Harris, 1997, Seismic attenuation tomography using the frequency shift method: Geophysics, 62, 895-905, doi: 10.1190/1 .1444197.

Robertsson, J. O., J. O. Blanch, and W. W. Symes, 1994, Viscoelastic finite-difference modeling: Geophysics, 59, 1444-1456, doi: 10.1190/1 .1443701 .

Stolt, R. H., and A. K. Benson, 1986, Seismic migration: Theory and practice: Geophysical Press.

Suh, S., K. Yoon, J. Cai, and B. Wang, 2012, Compensating visco-acoustic effects in anisotropic resverse-time migration: 82nd Annual International Meeting, SEG, Expanded Abstracts, doi: 10.1190/segam2012-1297.1.

Tang, Y., 2009, Target-oriented wave-equation least-squares migration/ inversion with phase-encoded Hessian: Geophysics, 74, no. 6, WCA95WCA107, doi: 10.1190/1.3204768.
Tarantola, A. 1984, Linearized inversion of seismic reflection data: Geophysical Prospecting, 32, 998-1015, doi: 10.1111/j.1365-2478.1984 .tb00751.x.

Valenciano, A., and N. Chemingui, 2013, Tomographic $Q$ estimation for viscoacoustic imaging: 83rd Annual Conference and Exhibition, EAGE, Extended Abstracts, Th0409.

Valenciano, A., N. Chemingui, D. Whitmore, and S. Brandsberg-Dahl, 2011, Wave equation migration with attenuation and anisotropy compensation: 81st Annual International Meeting, SEG, Expanded Abstracts, 232-236.

Wang, Y., 2008, Inverse Q-filtered migration: Geophysics, 73, no. 1, S1-S6, doi: $10.1190 / 1.2806924$

Whitmore, N., 1983, Iterative depth migration by backward time propagation: 53rd Annual International Meeting, SEG, Expanded Abstracts, 382385 .

Wong, M., S. Ronen, and B. Biondi, 2011, Least-squares reverse time migration/inversion for ocean bottom data: A case study: 81st Annual International Meeting, SEG, Expanded Abstracts, 2369-2373.

Xie, Y., K. Xin, J. Sun, C. Notfors, A. Biswal, and M. Balasubramaniam, 2009, 3D prestack depth migration with compensation for frequency dependent absorption and dispersion: 79th Annual International Meeting, SEG, Expanded Abstracts, 2919-2923.

Xin, K., B. Hung, S. Birdus, and J. Sun, 2008, 3D tomographic amplitude inversion for compensating amplitude attenuation in the overburden: 78th Annual International Meeting, SEG, Expanded Abstracts, 32393243.

Yu, Y., R. Lu, and M. Deal, 2002, Compensation for the effects of shallow gas attenuation with viscoacoustic wave equation migration: 72nd Annual International Meeting, SEG, Expanded Abstracts, 2062-2065.

Zhang, Y., P. Zhang, and H. Zhang, 2010, Compensating for visco-acoustic effects in reverse-time migration: 80th Annual International Meeting, SEG, Expanded Abstracts, 3160-3164.

Zhou, C., W. Cai, Y. Luo, G. T. Schuster, and S. Hassanzadeh, 1995, Acoustic wave-equation traveltime and waveform inversion of crosshole seismic data: Geophysics, 60, 765-773, doi: 10.1190/1.1443815. 